\title{
Review Article: Pitavastatin: Similarities and Differences Compared With Other Statins
}

\author{
Francesco $\mathrm{FICl}^{1}$, Bahar Arican TARIM², Gokhan FAIKOGLU³ , Elif Ilkay YUCE , Baris \\ GUNGOR $^{5}$, Nicolas Roberto ROBLES ${ }^{6}$ \\ 1 Catedra de Riesgo Cardiovascular, Universidad de Salamanca, Salamanca, Spain \\ 2 Department of Internal Medicine, Kartal Research and Education Hospital, Istanbul, Turkey \\ 3 Department of Medical Pharmacology, Beykent University- Beykent Faculty of Medicine, Istanbul, Turkey \\ 4 Department of Cardiology, Kelkit State Hospital, Gumushane, Turkey \\ 5 Department of Cardiology, University of Sciences and Research Hospital, Istanbul, Turkey 6 Hospital Universitario de \\ Badajoz. Badajoz. Spain
}

\begin{abstract}
Dyslipidemia is the leading cause of cardiovascular mortality and morbidity. Reduction of lipids, particularly low- density lipoprotein cholesterol (LDL-C), with statins, significantly decreases the risk of cardiovascular events. Among different statins, pitavastatin, exhibits a peculiar pharmacokinetic and pharmacological profile. Indeed, differently from other statins, pitavastatin: a) is not metabolized by hepatic cytochrome $\mathrm{CYP}_{3} \mathrm{~A}_{4}$ isoenzyme, therefore has a very low drug-drug interaction; b) has a similar or greater effect on LDL-C, c) is not associated with glucose metabolism impairment, and the risk of new onset diabetes is very low, d) increases high density lipoproteins (HDL) levels and, particularly, improves cholesterol efflux capacity of HDL, d) decreases cardiovascular outcome in primary and secondary prevention.
\end{abstract}

Keywords: Dyslipidemia, statins, pitavastatin, new-onset diabetes, HDL

Corresponding Autor:

Nicolas Roberto ROBLES

Hospital Universitario de Badajoz. Badajoz. Spain

nrrobles@yahoo.es

ORCID's:

Francesco Fici

: 000-0002-5366-7082

Bahar Arican

:0000-0002-6017-3259

Gokhan Faikoglu

: 0000-0002-8980-4499

Elif Bakır :0000-001-9208-7972

Baris Gungor

: 0000-0002-8883-117X

Nicolas Roberto Robles : 0000-003-4984-6691

(Received 08 February 2021, Accepted 3 June 2021) 


\section{INTRODUCTION}

Dyslipidemia remains the leading cause of cardiovascular (CV) morbidity and mortality and aggressive reduction of lipids significantly improve cardiovascular outcome. Reduction of low density lipoproteins cholesterol (LDL-C) remains the cornerstone for a significant prevention of cardiovascular events ${ }^{1,2}$. Lowering LDL-C by $2-3 \mathrm{mmol} / \mathrm{L}$ is associated with a significant $40 \%-50 \%$ risk reduction, also in patients with diabetes or at low (<10\%) CV risk ${ }^{1}$. Given the relationship between lipids lowering and CV protection, current guidelines recommend a LDL-C cut-off $<55 \mathrm{mg} / \mathrm{dl}$, (or at least $50 \%$ reduction, from baseline) and $<70 \mathrm{mg} / \mathrm{dL}$ (or at least 50\% reduction from baseline), in patients at very high or high CV risk respectively; while, in subjects at low-moderate risk, LDL$\mathrm{C}$ must be decreased to $<116$ and $100 \mathrm{mg} / \mathrm{dL}$ respectively ${ }^{3}$. Within the dyslipidemia treatment, statins are the most preferred medications. Therefore, this review mainly highlights the pharmacological, pharmacokinetic and therapeutic similarities and differences between the most prescribed pitavastatin and other available statins.

For this purpose, a literature search was conducted in PubMed, using the terms "pitavastatin", “dyslipidemia”, "new onset diabetes”, “adiponectin”, “ApoI” and "cardiovascular prevention", to identify eligible articles and review in English language, published in peer reviewed journal. We did not considered short communications, editorials and posters. The resulting articles were evaluated by the authors for suitability for this review.

\section{Pitavastatin and mechanism of action}

Pitavastatin, a new-generation lipophilic statin, is indicated for the treatment of primary and mixed dyslipidemia and also prevention of cardiovascular disease. The pharmacological mechanism is similar to that of other statins that is inhibition of the 3-hydroxy-3-methylglutaryl coenzyme A (HMG-CoA) reductase, with subsequent reduction of cholesterol synthesis.
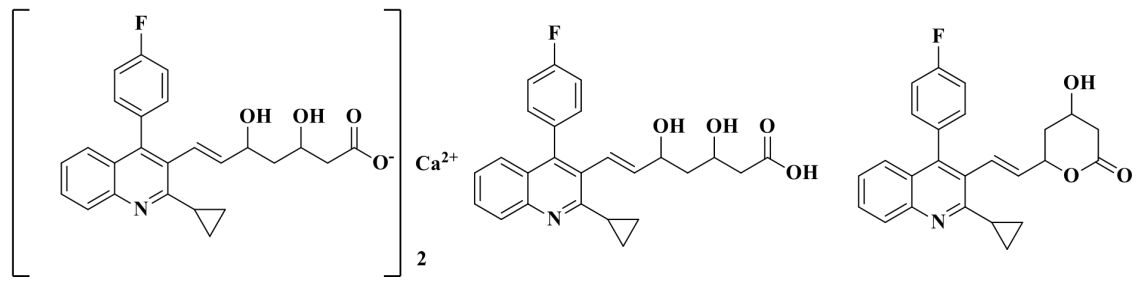

Figure 1: Pivastatin calcium salt and main metabolites ${ }^{4}$ 


\section{Pharmacokinetic and metabolic aspects}

Pitavastatin mainly inserted as calcium salt forms in pharmaceutical formulations. The quinoline ring and side chains that include fluorophenyl and cyclopropyl moieties provide improved pharmacokinetics, in the chemical structure of pitavastatin, in terms of lipid-water solubility balance. After oral administration, pitavastatin, is largely absorbed (80\%), with an absolute bioavailability $>60 \%$, higher than that of other lipophilic statins ${ }^{4}$. The peak plasma level is achieved after 0.5-1.2 h, without difference between single and multiple dos$\mathrm{es}^{4-6}$. Plasma concentrations and area under the curve (AUC) are proportional to the dose and reach the steady state after 4 days, without drug accumulation $^{4-6}$. The elimination half-life, after single and multiple dose, is 9-13 hours respectively. Pitavastatin is excreted unchanged in the bile and then reabsorbed through the enterohepatic circulation. This finding explains the long elimination half-life. The drug is minimally metabolized at hepatic level, where, through a process of glucuronidation, is converted to pitavastatin lactone, the main inactive metabolite, which in turn is reversibly reconverted in pitavastatin acid ${ }^{4,7}$. The excretion is in large part with the feces, while a very low amount $(<5 \%)$ is eliminated with urine, therefore dose reduction is not required in patients with kidney disease. (Figure1)

Differently from lovastatin, simvastatin, atorvastatin and fluvastatin, pitavastatin is not metabolized by hepatic cytochrome CYP3A4 isoenzyme, and, differently from rosuvastatin and fluvastatin, is minimally metabolized by $\mathrm{CYP} 2 \mathrm{C} 9$ isoenzymes, consequently the risk of clinically significant drugdrug interaction is very low ${ }^{4,6}$. This finding has relevant clinical implications, because, unlike other statins, avoid high plasma levels, when pitavastatin is co-administered with cardiovascular drugs, such as verapamil, diltiazem, digoxin, amiodarone, warfarin, clopidogrel or amlodipine ${ }^{6}$. Such characteristic improves tolerability and patient's adherence, particularly in case of polytherapy. The concomitant administration of pitastatin with ciclosporin is contraindicated, while the dose of pitavastatin must be reduced (1mg/daily) in patients taking erythromycin or clarithromycin ${ }^{6,7}$. Pharmacokinetic properties are not affected by food, does not differ between Caucasian and Asian, young and elderly subjects ${ }^{4,8,9}$. This is an important aspect, considering that subjects aged $\geq 65$ years have a higher prevalence of cardiovascular morbidity. 


\section{Therapeutic activity}

The lipid lowering of pitavastatin is either similar, or even greater than that of other statins, with a high prevalence of patients which achieve LDL-C target $^{10-15}$. (Figure 2)

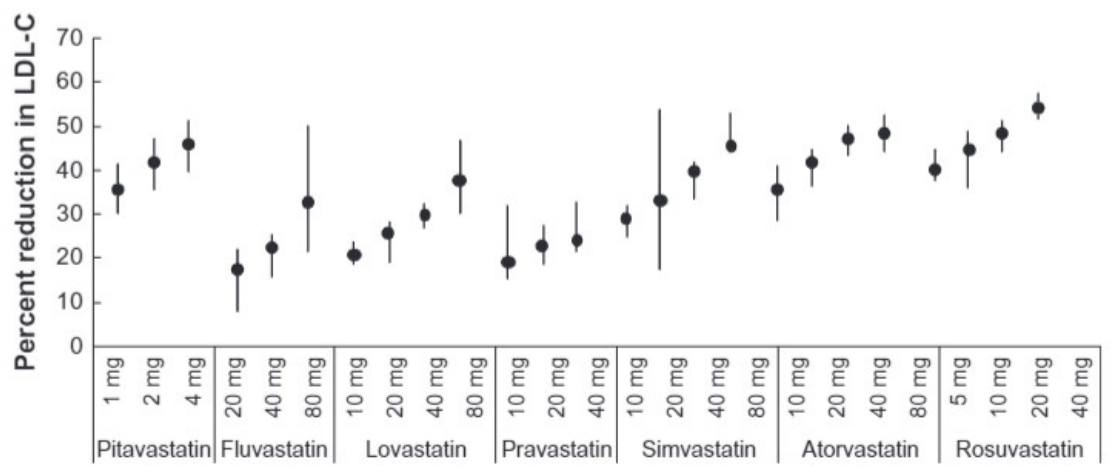

Figure 2: Comparison of percent reduction in LDL-C levels for different doses of statins. Taken from Saito Y Treatment Options for Hypercholesterolemia and Combined Dyslipidemia: Focus on Pitavastatin Clinical Medicine Insights: Therapeutics 2011:3 517-52510

Globally pitavastatin, decreases total cholesterol (TC 29\% -33\%), LDL-C, (42\% - 50\%), non-HDL-C (-41\%) and triglycerides (TG 30-32\%), according to the $\operatorname{dose}^{10,16,17}$. (Figure 3)

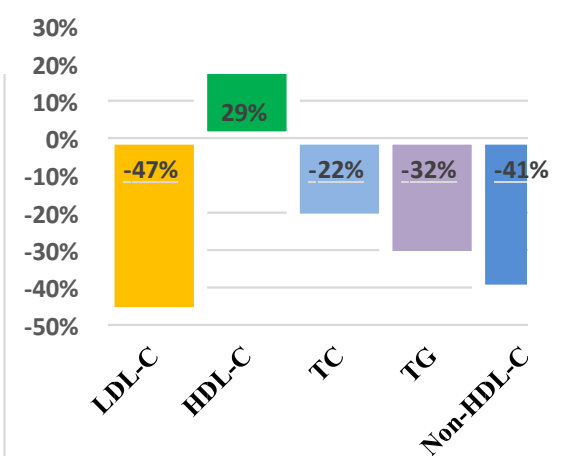

Figure 3: Effects of pitavastatin monotheraphy on lipid profile (HDL-C; High-density lipoprotein cholesterol, LDL-C; Ligh-density lipoprotein cholesterol, TC; Total cholesterol, TG; Total glyceride ${ }^{17}$ 
Compared to other statins, pitavastatin is about 6-fold more potent than atorvastatin, 1.7-fold more potent than rosuvastatin, 77-fold more potent than fluvastatin in reducing $\mathrm{LDL}-\mathrm{C}^{13}$.

Pitavastatin is equally effective, in elderly, in subjects with type 2 diabetes or metabolic syndrome and in people at high CV risk or with coronary artery disease $^{13,15,18-22}$.

However, pitavastatin, compared with other statins, differs in some pharmacological properties, concerning glucose metabolism and high-density lipoproteins (HDLs) plasma levels.

\section{Glucose metabolism}

The JUPITER trial raised concerns about the relationship between statins and new-onset diabetes (NOD), because $25 \%$ of patients treated with rosuvastatin developed $\mathrm{NOD}^{23}$. Moreover different meta-analyses and a recent cohort study, revealed that statins use is significantly associated with risk of $\mathrm{NOD}^{24-27}$. The incidence shows a remarkably variability (12\%-61.7\%), supporting the concept that, statin pharmacological properties, dosage, treatment duration and method to evaluate diabetes, play a major contributory role in the risk of NOD. Observational and comparative randomized clinical trials, have shown that pitavastatin, at variance of other statins, has a neutral, or even a favourable effect on glucose metabolism ${ }^{28,29}$.

Pitavastatin has been compared with other statins in patients with and without diabetes. The LIVESstudy subanalysis, performed in 1197 diabetic patients, untreated with antidiabetic drugs, revealed a significantly decrease of glycosylated hemoglobin (HbA1c), during 2 years of pitavastatin treatment ${ }^{30}$. The CHIBA study sub-analysis has shown that pitavastatin, differently from atorvastatin, did not increase glycoalbumin plasma levels and had a neutral effect on fasting plasma glucose, insulin and HOMA-IR ${ }^{31}$.

Furthermore a meta-analysis, involving non-diabetic patients shown that pitavastatin, compared with placebo or other statins, did not adversely affect glucose metabolism and decreased the risk of incident diabetes ${ }^{32}$.

These findings are in agreement with a, randomized, double-blind, controlled trial and particularly with the PATROL and PAPAGAO - T studies, which have shown that atorvastatin and rosuvastatin, differently from pitavastatin, have significantly increased HbA1c plasma concentration ${ }^{12,18,33}$. A retrospective study, compared pitavastatin with atorvastatin, pravastatin and pitavastatin in type 2 diabetic subjects with stable antidiabetic therapy ${ }^{34}$. Blood glucose and 
HbA1c increased with atorvastatin, but not with pitavastatin and pravastatin, suggesting the lack of difference between pravastatin and pitavastatin. However a recent meta-analysis, failed to show this similarity, because atorvastatin, rosuvastatin and also pravastatin, differently from pitavastatin, raised HbA1c and fasting blood glucose level ${ }^{35}$. Glicemic control during pitavastatin treatment was also evaluated in patients with coronary artery disease. In the Lamis II trial, no significant changes of HbA1c and blood glucose were observed in patients with acute myocardial infarct, after 1 year of treatment ${ }^{36}$. On the contrary pitavastatin significantly improved glucose metabolism in patients with acute coronary syndrome ${ }^{37}$. However some studies have reported a variable incidence of NOD during pitavastatin treatment. In the REAL-CAD trial incident diabetes was observed in $4.5 \%$ of patients with stable coronary artery disease, during 3 years follow-up and in patients at high risk of cardiovascular events NOD has been reported in $1.3 \%$ of subjects ${ }^{13,20}$. These results deserve some comments: a) both trials were large outcomes trials and were not adequately powered to assess the rate of NOD; b) in both studies, $42 \%$ and $20 \%$ of patients, respectively, were treated with beta-blockers, some of which are associated with a high risk of $\mathrm{NOD}^{38}$. However in patients with acute myocardial infarct, who did not have diabetes, the incidence of NOD with pitavastatin was $3 \%$, significantly lower than $8.4 \%$ and $10.4 \%$ observed with atorvastatin and rosuvastatin respectively ${ }^{39}$. This finding has been confirmed by a network metaanalysis and by a recent retrospective cohort study in nondiabetic patients, which indicate that the likelihood to develop diabetes was largest with atorvastatin, rosuvastatin, simvastatin, pravastatin and lovastatin, whereas it was significantly decreased with pitavastatin ${ }^{24,27}$.

Taken together, these findings show that pitavastatin, even at high doses, has a neutral effect on glucose metabolism, while the occurrence of new-onset diabetes cannot be ruled out, but the incidence rate is very limited. The pharmacological mechanism involved in this favourable aspect of pitavastatin is not yet fully understood. However accumulating evidence indicates that pitavastatin could stimulate adiponectin secretion, which, is involved in different biological process, such as insulin-sensitizing, anti-diabetic, anti-inflammatory and anti-atherosclerosis activities, through the increases fatty acid oxidation and glucose utilization in skeletal muscle and liver ${ }^{28,40}$. In spite of some discrepancies between different studies, pitavastatin, differently from other statins, significantly rises adiponectin plasma levels [27.2\%, vs $17.3 \%, 14.7 \%$ and $7.2 \%$ with rosuvastatin, pravastatin and atorvastatin respectively] $]^{28,41,42}$. 


\section{Effect on high-density lipoprotein cholesterol (HDL C)}

High plasma levels of HDLs seems to be related with low risk of CV events and, therefore, with atheroprotective properties ${ }^{43,44}$. Although statins usually lead to a minimal and variable change in HDLs, pitavastatin, differently from atorvastatin, pravastatin, fluvastatin and simvastatin, led to a significant increase in HDLs concentration (13.4\% -29.0\%), particularly in patients with low HDLs $(<40 \mathrm{mg} / \mathrm{dl})$ at baseline $\mathrm{e}^{1,30,42,45,46}$. (Figure 4)

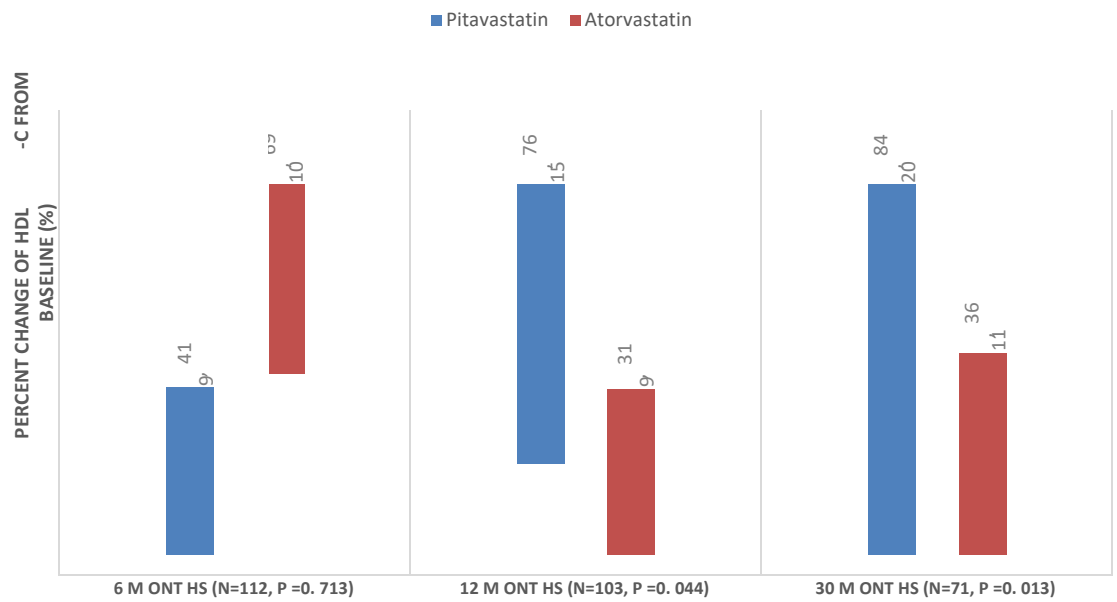

Figure 4: Time dependent percent change of HDL-C levels from baseline with Pitavastatin and Atorvastatin treatments ${ }^{46}$

Nevertheless, some concerns have been raised about the correlation between high HDLs level and CV protection, because pharmacological intervention, with drugs which increase HDLs, failed to show a significant reduction in CV outcome ${ }^{47,48}$.

There is evidence that HDLs, promote the "cholesterol efflux capacity" which correlates with the antiatherogenic effect of HDLs ${ }^{49,50}$.

Although a paucity of data, some studies provided evidence that pitavastatin, unlike other statins, was significantly associated with improved cholesterol efflux capacity of HDLs, [Fig 5 ] and suggest that such effect is mostly attributable to capacity of pitavastatin, differently from other statins, to increase apolipoprotein A1 plasma levels which improves HDL-C functionality through different biochemical mechanisms ${ }^{41,42,46,51 .}$ 


\section{Pitavastatin and coronary artery disease}

Statins are a mainstay in the primary and secondary prevention of atherosclerotic cardiovascular disease.

Different studies have assessed the therapeutic efficacy of pitavastatin in primary and secondary cardiovascular prevention. In patients with hypercholesterolemia and concomitant high cardiovascular risk factors, pitavastatin, compared with atorvastatin, provided a greater significant reduction of CV events (2.9\% vs $8.1 \%)$ and coronary revascularization for stable angina, $(4.5 \%$ vs $12.9 \%$ ), during 5 years of treatment ${ }^{13}$.

The REAL-CAD, a multicenter study, which involved 13054 patients with stable coronary artery disease, has shown that pitavastatin significantly decreased the risk of CV mortality and morbidity by $19 \%^{20}$. This result confirms the findings of the LAMIS and CIRCLE studies, performed in patients with acute myocardial infarct (AMI) ${ }^{21,52}$. Major cardiovascular events occurred in a small percentage of subjects (7.3\%-8.3\% respectively), lower than that observed with atorvastatin and pravastatin (19.3\% and 27.2\% respectively). However the risk of repeated coronary revascularization, either for new coronary lesions, or at target lesion was significantly reduced with pitavastatin compared to atorvastatin. In addition, in patients with acute coronary syndrome pitavastatin, was associated with stabilization of atherosclerotic plaque with increase fibrous-cap thickness and reduction of fibro-fatty volume index ${ }^{22,45,53,54}$. These effects were not inferior or significantly greater compared with atorvastatin ${ }^{22,53}$. Although in most studies high dose of pitavastatin, (4 mg/day), significantly protected patients from recurrent major CV event, the LAMIS II study (36) did not show significant difference between 2 and $4 \mathrm{mg}$ /day in patients with AMI (incidence of $\mathrm{CV}$ adverse events, $9.07 \%$ vs $9.13 \%$ respectively), confirming the results of LAMIS, CIRCLE and TOGETHAR studies, showing that even low dose of pitavastatin may decreases the incidence of major CV events, also in primary prevention13,21,36,45,52.

However, as the lipids lowering of pitavastatin is dose dependent, $4 \mathrm{mg} /$ daily, would be the suitable dosage for secondary CV prevention ${ }^{4,5}$.

\section{Future investigations}

While the role of pitavastatin in patients with dyslipidemia, associated or not with coronary artery disease, is well established, its efficacy in subjects with immunodeficiency virus (HIV) infections, deserve further investigations. However, the absence of pharmacokinetic interaction between antiretroviral drugs and pitavastatin, provides a strong rationale for a systemic use of pitavastatin 
to manage dyslipidemia and avoid CV outcome in these patients, The results of the REPRIEVE trial, now in progress will define the therapeutic place of pitavastatin in this group of patients $\mathrm{s}^{6,55,56}$.

\section{Safety and tolerability}

The most frequent adverse events (AEs) induced by statins are related to myopathy (in rare cases rhabdomyolysis) and liver injury.

Numerous studies have shown that pitavastatin is associated with a very low rate of AEs, also with high doses and during prolonged treatment ${ }^{20}$. Even if the rate of AEs differs across trials, overall 10.4\% of patients have reported AEs in the LIVES study, performed in approximately 20000 patients, treated with pitavastatin (1-4 mg/day) for 2 years ${ }^{57}$. The most common, mild in severity, AEs were myalgia, muscle spams or weakness, experienced by $1.08 \%, 0.18 \%$ of patients respectively. Overall, no clinically significant changes in laboratory parameters have been observed during the study. A mild increase of creatinine phosphokinase (CK), alanine aminotransferase (ALT), aspartate aminotransferase (AST) and $\gamma$-glutamyltransferase ( $\gamma$-GTP), incidence was found in $2,7 \%$, $1.8 \%, 1.5 \%$ and $1.0 \%$ of subjects respectively. Furthermore concomitant administration of pitavastatin with agents that, rising statins plasma levels, lead to AEs, was not associated with significant incidence of $\mathrm{AEs}^{8}$.

\section{RESULTS AND DISCUSSION}

Statins have become a cornerstone treatment in patients with dyslipidemia and in secondary prevention of atherosclerotic CV disease.

Pitavastatin, a new-generation lipophilic statin, indicated for the treatment of patients with dyslipidemia, shows similarities and differences compared with other statins.

Several studies have demonstrated that the lipid lowering of pitavastatin is similar, or even greater, than that of other statins, with a more significant effect in decreasing triglycerides and remnant lipoprotein cholesterol. The therapeutic efficacy has been documented in a wide range of patients with primary or combined dyslipidemia, also associated with type 2 diabetes or metabolic syndrome, in Asian and Caucasian subjects, in young and elderly patients, as well in subjects at high CV risk or with coronary artery disease. In this last group pitavastatin significantly decreased the rate of major adverse cardiovascular events and coronary plaque volume.

Unlike other statins, pitavastatin has a very low drug-drug interactions, because not metabolized by the $\mathrm{CYP}_{3} \mathrm{~A}_{4}$ pathways. This aspect has important clinical 
implications, because avoid the risk of high plasma level when co-administered with other cardiovascular drugs.

There is evidence that statins might increase the risk of NOD or may deteriorate glycemic homeostasis. Pitavastatin has a neutral or beneficial effect on glucose metabolism, by increasing adiponectin plasma levels and the incidence of NOD is very low, in comparison to other statins.

In addition, differently from some available stains, pitavastatin rises plasma levels of HDLs, improving their function, e.g the "reverse cholesterol efflux capacity" by stimulating the hepatic secretion of ApoA1.

Pitavastatin is well tolerated, also during long term administration, with very low incidence of dug related side effects, showing, in this way, a favorable riskbenefit profile.

\section{ETHICAL APPROVAL}

This article does not contain any studies with human participants or animals performed by any of the authors. 


\section{REFERENCES}

1. Baigent C, Blackwell L, Emberson J, Holland LE, Reith C, Bhala N, et al. Efficacy and safety of more intensive lowering of LDL cholesterol: A meta-analysis of data from 170000 participants in 26 randomised trials. The Lancet, 2o10; 376(9753): 1670-1681. doi:10.1016/ So1406736(10)61350-5

2. Navarese EP, Robinson JG, Kowalewski M, Koodziejczak M, Andreotti F, Bliden K, et al. Association between baseline LDL-C level and total and cardiovascular mortality after LDL-C lowering a systematic review and meta-analysis. JAMA, 2018; 319(15): 1566-1579. doi:10.1001/ jama.2018.2525

3. Mach F, Baigent C, Catapano AL, Koskinas KC, Casula M, Badimon L, Chapman MJ. (2020). 2019 ESC/EAS Guidelines for the management of dyslipidaemias: Lipid modification to reduce cardiovascular risk (Vol. 41, pp. 111-188).

4. Catapano AL. Pitavastatin - pharmacological profile from early phase studies. Atherosclerosis Supplements, 2010; 11(3): 3-7. doi:10.1016/S1567-5688(10)71063-1

5. Luo Z, Zhang Y, Gu J, Feng P, Wang Y. Pharmacokinetic properties of single- and multipledose pitavastatin calcium tablets in healthy chinese volunteers. Ther Res Clin Exp, 2015; 77: 52-57. doi:10.1016/j.curtheres.2015.02.001

6. Corsini A, Ceska R. Drug-drug interactions with statins: will pitavastatin overcome the statins' Achilles' heel?. Curr Med Res Opin, 2o11; 27(8): 1551-1562.doi:10.1185/03007995.2 011.589433

7. Hougaard Christensen MM, Bruun Haastrup M, Øhlenschlaeger T, Esbech P, Arnspang Pedersen S, Bach Dunvald AC, et al. Interaction potential between clarithromycin and individual statins-A systematic review, Basic Clin Pharmacol Toxicol, 2020; 126(4): 307-317. doi:10.1111/ bcpt.13343

8. Warrington S, Nagakawa S, Hounslow N. Comparison of the pharmacokinetics of pitavastatin by formulation and ethnic group: An open-label, single-dose, two-way crossover pharmacokinetic study in healthy caucasian and Japanese men. Clin Drug Investig, 2011; 31(10): 735-743. doi:10.2165/11592480-000000000-00000

9. Baker W, Chamberlin K. Benefit \risk assessment of pitavastatin for the treatment of hypercholesterolemia in older patients. Clin Interv Aging, 2015; 10: 733. doi:10.2147/CIA.S67532

10. Saito Y. Treatment options for hypercholesterolemia and combined dyslipidemia: Focus on pitavastatin. Clin Med Insights Ther, 2011; 3: 517-525.

11. Saku K, Zhang B, Noda K. Randomized head-to-head comparison of pitavastatin, atorvastatin, and rosuvastatin for safety and efficacy (quantity and quality of LDL) - The PATROL trialCirc, 2011; 75(6): 1493-1505. doi:10.1253/circj.CJ-10-1281

12. Moroi M, Nagayama D, Hara F, Saiki A, Shimizu K, Takahashi M, et al. Outcome of pitavastatin versus atorvastatin therapy in patients with hypercholesterolemia at high risk for atherosclerotic cardiovascular disease. Int J Cardiol, 2020; 305: 139-146. doi:10.1016/j.ijcard.2020.01.006

13. Adams SP, Alaeiilkhchi N, Wright JM. Pitavastatin for lowering lipids. Cochrane Database of Systematic Reviews, 2017; (7): 1-8. doi:10.1002/14651858.CDo12735

14. Sponseller CA, Morgan RE, Kryzhanovski VA, Campbell SE, Davidson MH. Comparison of the lipid-lowering effects of pitavastatin $4 \mathrm{mg}$ versus pravastatin $40 \mathrm{mg}$ in adults with primary 
hyperlipidemia or mixed (Combined) dyslipidemia: A phase IV, prospective, US, multicenter, randomized, double-blind, superiority trial. Clin Ther, 2014; 36(8): 1211-1222. doi:10.1016/j. clinthera.2014.06.009

15. Stender S, Budinski D, Gosho M, Hounslow N. Pitavastatin shows greater lipidlowering efficacy over 12 weeks than pravastatin in elderly patients with primary hypercholesterolaemia or combined (mixed) dyslipidaemia. Eur J Prev Cardiol, 2013; 20(1): 40-53. doi:10.1177/2047487312451251

16. Chan P, Shao L, Tomlinson B, Zhang Y, Liu ZM. An evaluation of pitavastatin for the treatment of hypercholesterolemia. Expert Opin Pharmacother, 2019; 20(1): 103-113. doi:10.1080 /14656566.2018.1544243

17. Tokgzoglu L, Zamorano JL. Current perspectives on the use of statins in the treatment of dyslipidaemic patients: Focus on pitavastatin. Drugs Context. 2020; 12 (9): $22020-4$ doi:10.7573/DIC.2020-4-4

18. Gumprecht J, Gosho M, Budinski D, Hounslow N. Comparative long-term efficacy and tolerability of pitavastatin $4 \mathrm{mg}$ and atorvastatin 20-40 $\mathrm{mg}$ in patients with type 2 diabetes mellitus and combined (mixed) dyslipidaemia. Diabetes, Obesity and Metabolism, 2011; 13(11): 10471055. doi:10.1111/j.1463-1326.2011.01477.x

19. Eriksson M, Budinski D, Hounslow N. Comparative efficacy of pitavastatin and simvastatin in high-risk patients: A randomized controlled trial. Adv Ther, 2011; 28(9): 811-823. doi:10.1007/s12325-011-0056-7

20. Taguchi I, Iimuro S, Iwata H, Takashima H, Abe M, Amiya E, et al. High-dose versus lowdose pitavastatin in Japanese patients with stable coronary artery disease (REAL-CAD) a randomized superiority trial. Circ, 2018; 137(19): 1997-2009.

21. Suh SY, Rha SW, Ahn TH, Shin EK, Choi CU, Oh DJ, et al. Long-term safety and efficacy of pitavastatin in patients with acute myocardial infarction (from the Livalo acute myocardial infarction study LAMIS ). Am J Cardiol, 2011; 108(11): 1530-1535. doi:10.1016/j.amjcard.2011.07.009

22. Hiro T, Kimura T, Morimoto T, Miyauchi K, Nakagawa Y, Yamagishi M, et al. Effect of Intensive Statin Therapy on Regression of Coronary Atherosclerosis in Patients With Acute Coronary Syndrome. A Multicenter Randomized Trial Evaluated by Volumetric Intravascular Ultrasound Using Pitavastatin Versus Atorvastatin. 2009; J Am Coll Cardiol, 54(4): 293-302. doi:10.1016/j.jacc.2009.04.033

23. Ridker PM, Danielson E, Fonseca FAH, Genest J, Gotto AM, Kastelein JJP, et al. Rosuvastatin to Prevent Vascular Events in Men and Women with Elevated C-Reactive Protein. NEJM, 2008; 359(21): 2195-2207. doi:10.1056/NEJMoa0807646

24. Thakker D, Nair S, Pagada A, Jamdade V, Malik A. Statin use and the risk of developing diabetes: a network meta-analysis. Pharmacoepidemiol Drug Saf, 2016; 25(10): 1131-1149. doi:10.1002/pds.4020

25. Casula M, Mozzanica F, Scotti L, Tragni E, Pirillo A, Corrao G, Catapano AL. Statin use and risk of new-onset diabetes: A meta-analysis of observational studies. Nutr Metab Cardiovasc Dis, 2017; 27(5): 396-406. doi:10.1016/j.numecd.2017.03.001

26. Navarese EP, Buffon A, Andreotti F, Kozinski M, Welton N, Fabiszak T, et al. Meta-analysis of impact of different types and doses of statins on new-onset diabetes mellitus. Am J Cardiol, 2013; 111(8): 1123-1130. doi:10.1016/j.amjcard.2012.12.037 
27. Na E, Cho S, Kim DJ, Choi J, Han E. Time-varying and dose-dependent effect of longterm statin use on risk of type 2 diabetes: A retrospective cohort study. Cardiovasc Diabetol, 2010;19(1). doi:10.1186/s12933-020-01037-0

28. Arnaboldi L, Corsini A. Could changes in adiponectin drive the effect of statins on the risk of new-onset diabetes? The case of pitavastatin. Atherosclerosis Supplements, 2015; 16: 1-27. doi:10.1016/S1567-5688(14)70002-9

29. Chapman MJ, Orsoni A, Robillard P, Hounslow N, Sponseller CA, Giral P. Effect of highdose pitavastatin on glucose homeostasis in patients at elevated risk of new-onset diabetes: Insights from the CAPITAIN and PREVAIL-US studies. Curr Med Res Opin, 2014; 30(5): 775784. doi:10.1185/03007995.2013.874989

30. Teramoto T, Shimano H, Yokote K, Urashima M. New evidence on pitavastatin: Efficacy and safety in clinical studies, Expert Opin Pharmacother, 2010; 11(5): 817-828.

31. Yokote K, Saito Y, Bujo H, Hanaoka H, Shinomiya M, Mikami K, et al. Influence of statins on glucose tolerance in patients with type 2 diabetes mellitus: Subanalysis of the collaborative study onhypercholesterolemia drug intervention and their benefits for atherosclerosis prevention (CHIBA Study). J Atheroscler Thromb, 2oo9; 16(3): 297-298. doi:10.5551/jat.E10o8

32. Vallejo-Vaz AJa. Effect of pitavastatin on glucose, HbA1c and incident diabetes: A metaanalysis of randomized controlled clinical trials in individuals without diabetes. Atherosclerosis, 2015; 241(2): 409-418. doi:10.1016/j.atherosclerosis.2015.06.001

33. Liu PY, Lin LY, Lin HJ, Hsia CH, Hung YR, Yeh HI, et al. Pitavastatin and Atorvastatin Double-Blind Randomized ComPArative Study among HiGh-Risk Patients, Including ThOse with Type 2 Diabetes Mellitus, in Taiwan (PAPAGO-T Study). PLoS ONE, 2013; 8(10), e.0076298. doi:10.1371/journal.pone.0076298

34. Yamakawa T, Takano T, Tanaka SI, Kadonosono K, Terauchi Y. Influence of pitavastatin on glucose tolerance in patients with type 2 diabetes mellitus. J Atheroscler Thromb, 20o8; 15(5): 269-275. doi:10.5551/jat.E562

35. Cui JY, Zhou RR, Han S, Wang TS, Wang LQ, Xie XH. Statin therapy on glycemic control in type 2 diabetic patients: A network meta-analysis. J Clin Pharm Ther, 2018; 43(4): 556-570. doi:10.1111/jcpt.12690

36. Hong YJ, Jeong MH, Bae JH, Oh SK, Rha SW, Hur SH, et al. Efficacy and safety of pitavastatins in patients with acute myocardial infarction: Livalo in Acute Myocardial Infarction Study (LAMIS) II. Korean Journal of Internal Medicine,2017; 32(4): 656-667. doi:10.3904/ kjim.2016.016

37. Wang YB, Fu XH, Gu XS, Fan WZ, Jiang YF, Hao GZ, et al. Effects of intensive pitavastatin therapy on glucose control in patients with non-ST elevation acute coronary syndrome. Am J Cardiol, 2017; 7(4): 89-96.

38. Bangalore S, Parkar S, Grossman E, Messerli FH. A Meta-Analysis of 94,492. Patients With Hypertension Treated With Beta Blockers to Determine the Risk of New-Onset Diabetes Mellitus. Am J Cardiol, 2007; 100(8):1254-62.

39. Choi SH, Lim S, Hong ES, Seo JA, Park CY, Noh, JH, et al. PROPIT: A PROspective comparative clinical study evaluating the efficacy and safety of PITavastatin in patients with metabolic syndrome. Clin Endocrinol, 2015; 82(5): 670-677. doi:10.1111/cen.12580

40. Drew BG, Duffy SJ, Formosa MF, Natoli AK, Henstridge DC, Penfold SA, et al. High-den- 
sity lipoprotein modulates glucose metabolism in patients with type 2 diabetes mellitus. Circ, 2009; 119(15): 2103-2111. doi:10.1161/CIRCULATIONAHA.108.843219

41. Sasaki J, Ikeda Y, Kuribayashi T, Kajiwara K, Biro S, Yamamoto K, et al. A 52Week, Randomized, Open-Label, Parallel-Group Comparison of the Tolerability and Effects of Pitavastatin and Atorvastatin on High-Density Lipoprotein Cholesterol Levels and Glucose Metabolism in Japanese Patients with Elevated Levels of Low-Density Li. Clin Ther, 2008; 30(6): 1089-1101. doi:10.1016/j.clinthera.2008.05.017

42. Pirillo A, Catapano AL. Pitavastatin and HDL: Effects on plasma levels and function(s). Atheroscler Suppl, 2017; 27: e1-e9.

43. Nakazawa M, Arashi H, Yamaguchi J, Ogawa H, Hagiwara N. Lower levels of highdensity lipoprotein cholesterol are associated with increased cardiovascular events in patients with acute coronary syndrome. Atherosclerosis, 2020; 303: 21-28. doi:10.1016/j.atherosclerosis.2020.05.005

44. Acharjee S, Boden WE, Hartigan PM, Teo KK, Maron DJ, Sedlis SP, et al. Low levels of highdensity lipoprotein cholesterol and increased risk of cardiovascular events in stable ischemic heart disease patients: A post-hoc analysis from the COURAGE trial (clinical outcomes utilizing revascularization and aggressive drug evaluat. J Am Coll Cardiol, 2013; 62(20): 1826-1833. doi:10.1016/j.jacc.2013.07.051

45. Kodama K, Komatsu S, Ueda Y, Takayama T, Yajima J, Nanto S, et al. Stabilization and regression of coronary plaques treated with pitavastatin proven by angioscopy and intravascular ultrasound: The TOGETHAR trial. Circ, 2010; 74(9): 1922-1928. doi:10.1253/circj.CJ-10-0038

46. Kurogi K, Sugiyama S, Sakamoto K, Tayama S., Nakamura S, Biwa T, et al. Comparison of pitavastatin with atorvastatin in increasing HDL-cholesterol and adiponectin in patients with dyslipidemia and coronary artery disease: The COMPACT-CAD study. J Cardiol, 2013; 62(2): 87-94. doi:10.1016/j.jjcc.2013.03.008

47. Niacin in Patients with Low HDL Cholesterol Levels Receiving Intensive Statin Therapy. NEJM, 2011; 365(24): 2255-2267. doi:10.1056/nejmoa1107579

48. Schwartz GG, Olsson AG, Abt M, Ballantyne CM, Barter PJ, Brumm J, et al. Effects of Dalcetrapib in Patients with a Recent Acute Coronary Syndrome. NEJM, 2012; 367(22): 20892099. doi:10.1056/nejmoa1206797

49. Khera AV, Cuchel M, de la Llera-Moya M, Rodrigues A, Burke MF, Jafri K, et al. Cholesterol Efflux Capacity, High-Density Lipoprotein Function, and Atherosclerosis. NEJM, 2o11; 364(2): 127-135. doi:10.1056/nejmoa1001689

50. Rohatgi A, Khera A, Berry JD, Givens EG, Ayers CR, Wedin KE, et al. HDL Cholesterol Efflux Capacity and Incident Cardiovascular Events. NEJM, 2014; 371(25): 2383-2393. doi:10.1056/ nejmoa1409065

51. Miyamoto-Sasaki M, Yasuda T, Monguchi T, Nakajima H, Mori K, Toh R, Ishida T, Hirata KI. Pitavastatin Increases HDL Particles Functionally Preserved with Cholesterol Efflux Capacity and Antioxidative Actions in Dyslipidemic Patients. J Atheroscler Thromb. 2013; 20 (9): 708-716.

52. Maruyama T, Takada M, Nishibori Y, Fujita K, Miki K, Masuda S, et al. Comparison of preventive effect on cardiovascular events with different statins: The circle study. Circ, 2o11; 75(8): 1951-1959. doi:10.1253/circj.CJ-10-1163 
53. Toi T, Taguchi I, Yoneda S, Kageyama M, Kikuchi A, Tokura M, et al. Early effect of lipidlowering therapy with pitavastatin on regression of coronary atherosclerotic plaque - Comparison with atorvastatin. Circ, 2009; 73(8): 1466-1472. doi:10.1253/circj.CJ-08-1051.

54. Nishiguchi T, Kubo T, Tanimoto T, Ino Y, Matsuo Y, Yamano T, et al. Effect of Early Pitavastatin Therapy on Coronary Fibrous-Cap Thickness Assessed by Optical Coherence Tomography in Patients With Acute Coronary Syndrome: The ESCORT Study. JACC: Cardiovascular Imaging, 2018; 11(6): 829-838. doi:10.1016/j.jcmg.2017.07.011

55. Malvestutto CD, Ma Q, Morse GD, Underberg JA, Aberg JA. Lack of pharmacokinetic interactions between pitavastatin and efavirenz or darunavir/ritonavir. J Acquir Immune Defic Synd, 2014; 67(4): 390-396. doi:10.1097/QAI.0000000000000333

56. Gilbert JM, Fitch KV, Grinspoon SK. HIV-related cardiovascular disease, statins, and the REPRIEVE trial. Top antivir med, 2015; 23(4): 146-149.

57. Kurihara Y, Douzono T, Kawakita K, Nagasaka Y. A Large-scale, Long-term, Prospective Post-marketing Surveillance of Pitavastatin. 薬理と治療, 20o8; 36(8): 709-731. 